\title{
Multimodal Low-Dimensional Materials Characterization with Correlative Microscopy: Raman-PL-FLIM-AFM-SNOM-SEM
}

\author{
Ute Schmidt ${ }^{1}$, Wei Liu ${ }^{2}$, David Steinmetz ${ }^{1}$, Thomas Dieing ${ }^{1}$, and Olaf Hollricher ${ }^{1}$ \\ 1. WITec GmbH, Ulm, Germany (www.witec.de) \\ 2. WITec Instruments, Knoxville, TN, USA
}

The characterization of low-dimensional materials such as graphene or transition metal dichalogenides (TMDCs) often requires more than one technique to obtain a thorough understanding of their attributes for specific applications. Graphene and TMDCs both have layered structures and properties that vary significantly with thickness as compared to single layer conformations, making them very interesting for electronics design [1]. Their photonic and optoelectronic qualities have been shown to enable ultrafast carrier dynamics and ultra-sensitivity in UV-VIS, IR and THz frequency ranges [2]. Electronic device performance optimization can benefit greatly from knowledge of their crystalline structure and exciton dynamics. The aim of the following analysis is to show how several spectroscopy (Raman/Photoluminescence) and microscopy techniques (AFM/SEM) can, in correlation, provide a more richly detailed depiction of low-dimensional materials than the constituent measurements could offer in isolation.

Raman spectroscopy, and Raman imaging in particular, has proved to be of great value in differentiating spectra obtained from single, double and multi-layered low-dimensional materials. Raman imaging was also used to evaluate strain, doping, chirality and disorder in graphene [3]. The information acquired with Raman spectroscopy and imaging can be complemented by data from other techniques such as Atomic Force Microscopy (AFM), Scanning Nearfield Optical Microscopy (SNOM), current sensing, Scanning Electron Microscopy (SEM) and measurements performed at low temperatures $(<10 \mathrm{~K})$ and under strong magnetic fields (up to 9T).

The two-dimensional forms of TMDCs are also often characterized using the same experimental methods. Fig. 1 shows an example of a correlative AFM-PL-Raman-FLIM measurement of CVD-grown $\mathrm{WS}_{2}$ crystal on a $\mathrm{Si} / \mathrm{SiO}_{2}$ substrate. For this group of low-dimensional materials the information obtained through correlation was revelatory as some show photoluminescence (PL) only in single layers, which can be measured with exceptionally high resolution using SNOM-PL. Furthermore, using RISE microscopy (a combination of Raman imaging and SEM) allows the characterization of these sensitive materials inside the vacuum chamber without exposure to air. Modifications performed using the electron beam will also be described.

\section{References:}

[1] A. K. Geim , I. V. Grigorieva, Nature 499 (2013), p. 419.

[2] F. H. L. Koppens, T. Nueller, P. Avouris, A.C. Ferrari, M. S. Vitiello, M. Polini, M. Nat. Nano 9 (2014) p. 780.

[3] Y. You, Z. Ni, T.Yu, Z. Shen, Appl. Phys. Letters 39 (2008) p.13112. 

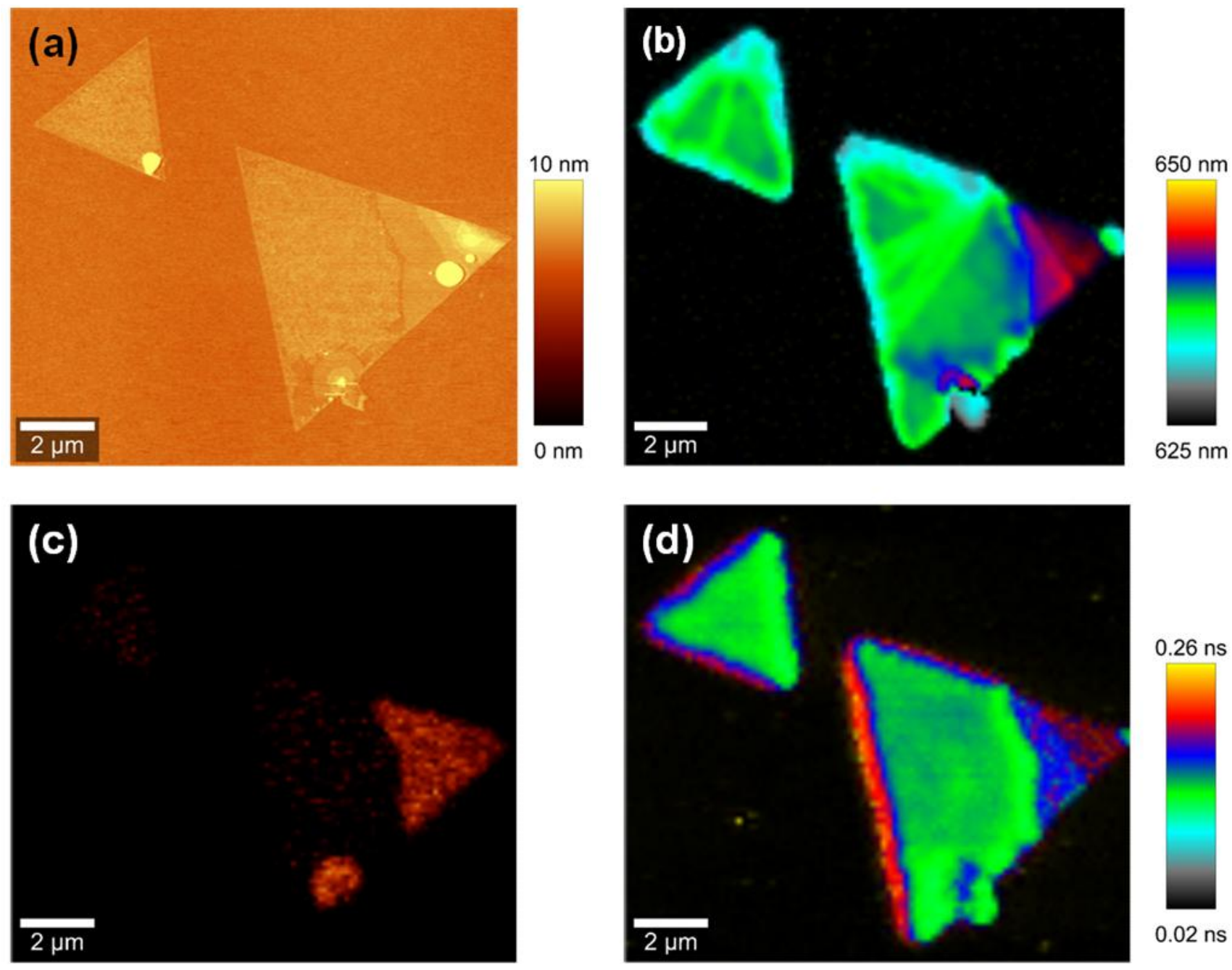

$625 \mathrm{~nm}$

Figure 1. (a) AFM topography image of a $2 \mathrm{D}-\mathrm{WS}_{2}$ crystal CVD-grown on a $\mathrm{SiO}_{2} / \mathrm{Si}$ substrate. (b) Correlated PL image showing the position of the emission peak, clearly showing the layer-dependent band-gap.

(c) Correlated Raman image showing the peak intensity at $27 \mathrm{~cm}^{-1}$, revealing the weak phonon interaction between layers. (d) Correlated FLIM image based on the PL in (b), showing different exciton lifetimes at different layers and structural edges. 